\title{
Influence of geometrical variations on morphodynamic equilibria in short tidal basins
}

\author{
Corine Meerman ${ }^{1}$ (D) . Vivi Rottschäfer ${ }^{1} \cdot$ Henk Schuttelaars $^{2}$
}

Received: 6 April 2017 / Accepted: 2 November 2018 / Published online: 13 December 2018

(C) The Author(s) 2018

\begin{abstract}
The existence of cross-sectionally averaged morphodynamic equilibria of tidal inlets is investigated, using a crosssectionally averaged model, and their sensitivity to variations of geometry, deposition parameter, frictional effects and advective sediment transport is analysed. Different geometries, from exponentially converging to exponentially diverging, are considered for inlets with lengths typical for the Dutch Wadden Sea. Standard continuation techniques are employed to numerically obtain morphodynamic equilibrium solutions, i.e. solutions for which the tidally averaged bed level does not change anymore. It is known that when the water motion at the entrance of the inlet is only forced by a $M_{2}$ tidal constituent assuming the water level to be spatially uniform and only diffusive sediment transport is considered, the morphodynamic bed equilibrium has a constantly sloping profile for a rectangular inlet. We find that the bed profile in equilibrium becomes convex (concave) when we change the frictionless embayment geometry to a diverging (converging) geometry. Upon letting the deposition parameter depend on the depth, a more convex bed profile for all geometries considered is found. Including frictional effects in the momentum equation has a minor effect when only diffusion is considered, but the bed profile changes significantly when advection is included. When the tidal forcing of the sea surface elevation depends on a $M_{4}$ tidal constituent as well, the morphodynamic equilibrium bed varies from very deep to shallow, depending on the relative phase. For a diverging inlet geometry, there are combinations of the relative phase and tidal basin length for which we show the existence of multiple equilibria. This implies that for these geometries, the cross-sectionally averaged bed profile in morphodynamic equilibrium can change significantly when the relative phase or the embayment length is changed. The magnitude of the perturbation necessary to actually evolve towards the other equilibrium and the time scale associated with this change can not be inferred from the analysis presented in this paper.
\end{abstract}

Keywords Tidal basin $\cdot$ Idealised model $\cdot$ Morphodynamic equilibrium $\cdot$ Sediment transport $\cdot$ Multiple equilibria

\section{Introduction}

A barrier coast consists of several barrier islands with a tidal inlet between these islands connecting one or more back

This article is part of the Topical Collection on the 18th conference on Physics of Estuaries and Coastal Seas (PECS), Scheveningen, Netherlands, 9-14 October 2016

Responsible Editor: Mick van der Wegen

Corine Meerman

cmeerman@math.leidenuniv.nl

1 Mathematical Institute, Leiden University, P.O. Box 9512, 2300 RA Leiden, The Netherlands

2 Delft Institute of Applied Mathematics, Delft University of Technology, Mourik Broekmanweg 6, 2628 XE, Delft, The Netherlands barrier basins to the sea or ocean. This type of coastal feature occurs at approximately $10 \%$ of world's coastline, Glaeser (1978). Large parts of these basins fall dry during a part of the tidal cycle, which makes them important for ecological, economical and recreational purposes. To manage these different interests, it is essential to obtain a better understanding of these systems and their sensitivity to natural and human interference.

Already quite some research has been conducted on this topic. Both the water motion and the morphodynamic evolution in shallow tidal inlet systems have been studied extensively. The linear dynamics of the tidal motion was first studied by Green (1837). It was shown in Parker (1991) and Zimmerman (1981) that nonlinear interactions result in the generation of overtides and residual currents. In the last decades, many analytic solutions have been presented for the tidal motion in a wide range of estuarine geometries, 
see Friedrichs (2010) for a review. In the studies mentioned above, no feedback to morphology was considered. When taking the interaction of the currents with the erodible bed into account, complex patterns can develop, see De Swart and Zimmerman (2009). A lot of research has been conducted on the sensitivity of these bed patterns to various physical parameters. The influence of sediment supply on morphodynamic bed equilibria was studied by Van der Wegen et al. (2017), Maan et al. (2015) and Robert et al. (2000), the influence of frictional effects by Lanzoni and Seminara (1998, 2002), and Schuttelaars and de Swart (2000), the influence of including mud in the sediment transport by Van Ledden et al. (2004) and the influence of geometry by Lanzoni and Seminara $(1998,2002)$ and Van Leeuwen et al. (2000).

Although many studies have investigated a similar topic, one has to take care in directly comparing the results, because the studies often use different models and solution methods. For a classification of these different types of morphodynamic models, see De Vriend and Ribberink (1996) and Murray (2013). Here, we restrict ourselves to cross-sectionally averaged idealised processbased models, which are mathematical models based on first physical principles. The equations are simplified such that only those processes are taken into account which, according to a detailed scaling analysis, are important. Note that by averaging the models over the width, observed channel-shoal patterns cannot be reproduced, only crosssectionally averaged quantities are found. However, a good understanding of cross-sectionally averaged equilibria is essential as the first step in a depth-averaged (linear) stability approach that can be used to unravel the initial formation of channel-shoal patterns and the resulting finite-amplitude patterns (see Dijkstra et al. (2014)). This approach implies that the influence of tidal flats which are shown to be important by Ridderinkhof et al. (2016) and Van Prooijen and Wang (2013) is not parametrically included in the width-averaged model, but only starts to play an important role as tidal flats are formed in a $2 \mathrm{DH}$ analysis.

In Van Leeuwen et al. (2000), the authors used such an idealised model to analyse geometric variations of the embayment on the morphodynamic bed profiles. The sea surface elevation was assumed to be spatially uniform and the sediment concentration was given by an advectiondiffusion equation. When the sea surface elevation was only forced by a $M_{2}$ tidal constituent and diffusive sediment transport was assumed to be dominant, the authors found that the morphodynamic bed profile becomes more convex as the width convergence increases. When the sea surface elevation was forced by both a $M_{2}$ and a $M_{4}$ tidal constituent and a converging embayment, there was a maximum length for the embayment for which a morphodynamic equilibrium could be found, for a relative phase difference $\phi$ between
$M_{2}$ and $M_{4}$ of $\phi \in\left[0^{\circ}, 180^{\circ}\right]$. Letting the deposition parameter depend on the depth, the authors found that the equilibrium bed profiles became more convex.

Instead of fixing the bed at the landward boundary, Lanzoni and Seminara (2002) created an inner boundary condition at the landward side to allow for wetting and drying. Using this approach the authors defined the length of an embayment as the maximum length for which a morphodynamic equilibrium still exists. These results were confirmed by Todeschini et al. (2008). From these studies, it was concluded that the maximum length of the embayment was mainly governed by the convergence length, although frictional effects also influenced the maximum length for weakly convergent embayments.

In Van Leeuwen et al. (2000), and Lanzoni and Seminara $(1998,2002)$, the morphodynamic equilibria were found by time-integration. A different way of solving a morphodynamic model is to make use of a fixed point seeker. This method was first used in Schuttelaars and de Swart (1996). In their model, the authors neglected effects of waves, density currents, inertia and friction and the sea surface elevation was assumed to be spatially uniform. The authors considered a rectangular embayment with a fixed bed at the seaward and landward boundaries and assumed the deposition parameter to be spatially constant. The authors performed a systematic analysis of the different types of sediment transport. Considering only a prescribed $M_{2}$ tidal forcing at the entrance, a unique morphodynamic equilibrium was found with a constantly sloping bed. When the sea surface elevation at the entrance was forced by both a $M_{2}$ and $M_{4}$ tidal constituent, the bed profile became either convex $\left(0^{\circ}<\phi<180^{\circ}\right)$ or concave $\left(180^{\circ}<\phi<360^{\circ}\right)$. Schuttelaars and de Swart (2000) extended this analysis to embayments of arbitrary length, including bottom friction and inertia. The existence of multiple stable equilibria was shown for long enough embayments, when the water motion was forced by both a $M_{2}$ tidal constituent and a strong enough $M_{4}$ constituent. These results were confirmed in Hibma et al. (2003) using a numerical simulation model.

In Ter Brake and Schuttelaars (2010), the authors extended the idealised model of Schuttelaars and de Swart (1996) by including a topographically induced transport term in the bed evolution equation. The authors also analysed the influence of different boundary conditions on the bed. The sea surface elevation was again assumed to be uniform and the geometry of the embayment was considered to be rectangular.

The research presented in this paper extends the model of Ter Brake and Schuttelaars (2010) by including inertial and frictional effects in the momentum equation and allowing for geometrical variations in the embayment. The channelshoal structure of observed patterns develop as instabilities on the width-averaged morphodynamic equilibria. In this 
article, these width-averaged morphodynamic equilibria are identified. The goal of this article is to analyse the sensitivity of width-averaged morphodynamic equilibria in a rectangular, exponentially converging and exponentially diverging embayment for the following physical parameters: inertia, bed shear stress, depth-dependence of the deposition parameter, inclusion of advective processes and inclusion of an externally prescribed overtide. To validate the model, results are qualitatively compared to profiles obtained from observations.

The outline of this article is as follows. In Section 2, the model geometry and equations are presented. In Section 3 the full model equations are scaled and analysed using an asymptotic expansion. The effect of choosing various geometries for the embayment when only considering diffusive processes is analysed in Section 4. In this section, the influence of the formulation of the deposition parameter, the friction and the inclusion of advective sediment transport for different geometries is also studied. Furthermore, the existence of multiple equilibria is discussed. Conclusions are given in Section 5.

\section{The model}

\subsection{The geometry of the embayment}

The geometry of the embayment that we consider has a prescribed length $L$ and a prescribed width $W$ that is allowed to vary as a function of the longitudinal coordinate $x$, see Fig. 1. The embayment has three non-erodible coastlines, an open connection to the sea at $x=0$ and a bed that is erodible. The sea surface elevation is denoted by $z=\zeta$, the bottom is located at $z=-H+h$ which results in a local water depth given by $H-h+\zeta$, with $H$ the undisturbed water depth. The landward boundary is located at the intersection of the bed and the sea surface elevation. Due to the tidally varying sea surface elevations, the landward boundary is actually a moving boundary and is denoted by $\hat{x}$. The length of the embayment is defined such that $\langle\hat{x}\rangle=L$, with $\langle\cdot\rangle$ representing tidal averaging.

\subsection{Modelling approach}

In order to find morphodynamic equilibria, we construct a model that describes the complex interaction between the water motion, sediment transport and bed evolution. The water motion is described by the shallow water equations and transport of suspended sediment is described by an advection-diffusion equation. The bed evolves due to convergences and divergences of suspended load transport, resulting from erosion of the bed and deposition of suspended material and bed load transport. When the bed is steady over the long morphodynamic timescale, a morphodynamic equilibrium is said to be obtained.

\subsection{The water motion}

We consider embayments, representative for those observed in the Wadden Sea, with lengths between $10 \mathrm{~km}$ and $20 \mathrm{~km}$, widths varying between $0.5 \mathrm{~km}$ and $19 \mathrm{~km}$, and depths $\sim 10 \mathrm{~m}$. Hence, the embayment is shallow $(H \ll L)$. In this case, the water motion can be described by the depthintegrated and width-averaged shallow water equations for a homogeneous fluid, see Csanady (1982). When the influence of wind and waves is not taken into account and density differences are neglected, the continuity equation and the momentum equation are given by

$$
\begin{aligned}
& W \zeta_{t}+[W(\zeta+H-h) u]_{x}=0, \\
& u_{t}+u u_{x}+\frac{\tau_{\text {bed,x }}}{\rho(H-h+\zeta)}=-g \zeta_{x} .
\end{aligned}
$$

Here, $u$ is the depth-averaged and width-averaged water velocity, $g$ denotes the gravitational acceleration, and $\rho$ the density of water. We use a linearised formulation for the bottom friction term, (Zimmerman 1992):

$\tau_{\text {bed,x }}=\rho r u$.

Here, $r$ the bottom friction coefficient, which is given by $r=\frac{8 U c_{d}}{3 \pi}$ with $U$ a characteristic velocity scale and $c_{d}$ the drag coefficient. At the seaward side, the sea surface elevation $\zeta$ is prescribed as a combination of the $M_{2}$ tidal constituent and its first overtide $M_{4}$. We assume the water depth to vanish at the landward boundary. This leads to the boundary conditions

$$
\begin{array}{lr}
\zeta=A_{M_{2}} \cos \left(\sigma t-\phi_{M_{2}}\right)+A_{M_{4}} \cos \left(2 \sigma t-\phi_{M_{4}}\right) & \text { at } x=0, \\
u=\hat{x}_{t} & \text { at } x=\hat{x},
\end{array}
$$

where the frequency of the principal tide is given by $\sigma=$ $\frac{2 \pi}{T}$, with $T$ the tidal period of the $M_{2}$-tidal constituent. Here, $A_{M_{2}}$ and $A_{M_{4}}$ are the tidal amplitudes of the $M_{2}$ and $M_{4}$ tidal constituents at the entrance of the embayment, and $\phi_{M_{2}}$ and $\phi_{M_{4}}$ their corresponding phases.

\subsection{Suspended sediment transport}

To describe the sediment transport, the concentration equation is integrated over the depth and averaged over the width, see Van Rijn (1993),

$W C_{t}+[W C u]_{x}-\kappa_{h}\left(W C_{x}\right)_{x}=W \alpha u^{2}-\frac{\omega_{s}^{2}}{\kappa_{v}} \beta W C$,

with $C$ the depth-integrated and width-averaged sediment concentration with dimension $\mathrm{kg} / \mathrm{m}^{2}, \kappa_{h}$ the horizontal diffusivity, $\kappa_{v}$ the vertical diffusivity, $\omega_{s}$ the settling velocity and $\alpha$ a sediment erosion coefficient. The first term on the 
Fig. 1 A sketch of the geometry used. a A top view of the embayment with a rectangular (solid), converging (dotted) or diverging (dashed) width profile. $\mathbf{b}$ A cross-sectionally view of the embayment

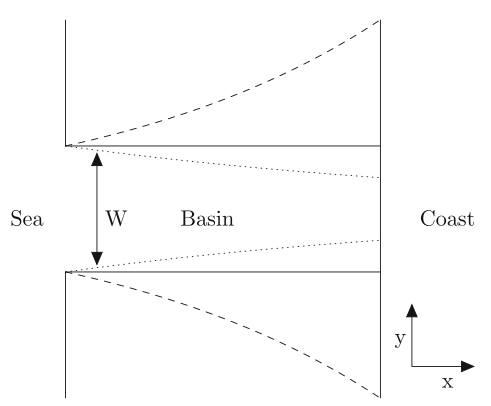

(a)

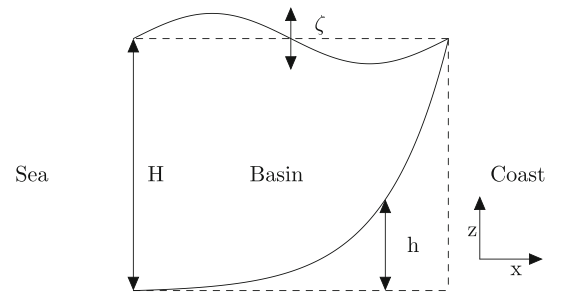

(b) right-hand side models the whirling up of sediment from the bed and the second term the deposition of sediment. On the left-hand side, the temporal changes of the concentration are described by the first term, the second term denotes the divergence of the advective sediment transport and the remaining term models the diffusive contribution. Following Ter Brake and Schuttelaars (2010), we take the erosion coefficient $\alpha$ as

$\alpha=\rho_{s}(1-p) \frac{\Gamma \omega_{s}}{u_{c}^{2}}$,

with $\rho_{s}$ the density of the sediment, $p$ the bed porosity, $u_{c}$ the critical depth-averaged friction velocity for erosion and $\Gamma$ an empirical constant (Smith and McLean 1977; Van Leeuwen 2002). Moreover, similar to Ter Brake and Schuttelaars (2010) and Van Leeuwen et al. (2000), we let the deposition parameter depend exponentially on the local water depth:

$\beta=\left(1-e^{-\frac{\omega_{s}}{\kappa_{v}}(H-h+\zeta)}\right)^{-1}$.

Since we are interested in morphodynamic equilibria, we impose at the seaward side the condition that the tidally averaged bed level does not change, implying a balance between tidally averaged sediment erosion and deposition. At the moving landward boundary no residual sediment transport is allowed. This leads to

$$
\begin{aligned}
\left\langle\alpha u^{2}-\frac{\omega_{s}^{2}}{\kappa_{v}} \beta C\right\rangle & =0 \quad \text { at } \quad x=0, \\
-\kappa_{h}\left\langle W C_{x}\right\rangle & =0 \quad \text { at } \quad x=\hat{x} .
\end{aligned}
$$

Note that there is no sediment transport due to advection at $x=\hat{x}$ because we consider a moving boundary. Therefore, only the diffusive part has to be zero. The sediment concentration can be decomposed into a fluctuating and a residual part, $C=\hat{C}+<C>$, with $<\hat{C}>=0$. Note that there is the possibility that a diffusive boundary layer develops at one of the boundaries in the fluctuating part of the concentration, see Van Leeuwen et al. (2000). To avoid these temporally fluctuating boundary conditions, we assume that, in the limit of $\kappa_{h} \rightarrow 0$, the fluctuating part of the solution of the sediment concentration equation agrees with the solution for $\kappa_{h}=0$. Hence, for the fluctuating part of the sediment transport, the boundary conditions are given by

$\lim _{\kappa_{h} \rightarrow 0} \hat{C}\left(x, t, \kappa_{h}\right)=\hat{C}\left(x, t, \kappa_{h}=0\right) \quad$ at $x=0$ and $x=\hat{x}$.

\subsection{The bed evolution equation}

The bed evolution is described by considering the net effect of sediment erosion and deposition during one tidal cycle,

$W \rho_{s}(1-p) h_{t}=\rho_{s}(1-p) \mu\left(W h_{x}\right)_{x}-\left\langle W\left(\alpha u^{2}-\frac{\omega_{s}^{2}}{\kappa_{v}} \beta C\right)\right\rangle$,

with $\mu$ the magnitude of the bedload transport related to bed slope effects. The first term on the right-hand side of Eq. 2.1 represents the diffusive bedload transport related to bed slope effects. The other terms model the local erosion and deposition of sediment, respectively. At the seaward boundary, we require that the bottom is fixed and at the moving landward boundary, the undisturbed water depth is zero,

$h=0 \quad$ at $x=0$,

$h=H-h+\zeta \quad$ at $\quad x=\hat{x}$.

Since we are looking for equilibrium solutions, a boundary condition at the entrance has to be prescribed for the depth. Here, we choose to prescribe the depth of the tidal basin system at the entrance Ter Brake and Schuttelaars (2010). For a detailed discussion on the choice of the boundary condition at the entrance, we refer to Ter Brake and Schuttelaars (2010) where it is shown how morphodynamic equilibria which result from imposing a boundary condition at the entrance, different from the one used in this paper, can be obtained from the equilibria found with a prescribed depth at the entrance. As shown in Ter Brake and Schuttelaars (2010), using a boundary condition different from fixing the depth at the entrance, results in morphodynamic equilibria with an a priori unknown depth at the entrance (i.e. the depth at the entrance cannot 
be chosen arbitrarily when the length of the system is prescribed). In this paper, we will focus on morphodynamic equilibria for embayments with a prescribed length and a fixed depth at the entrance (which is consistent with requiring $h=0$ at $x=0$ ), where one can think of the prescribed length and entrance depth as coming from observations (see Section 4.4 for two examples). This implies that we try to find morphodynamic equilibria, given the length of the system and the depth at the entrance. Transient behaviour can not be captured using this type of models.

\section{Solution method}

\subsection{Scaling the model}

To develop an idealised model that contains only the most essential processes, we need to assess the importance of the various terms in the equations. Therefore, we introduce dimensionless variables by scaling the dimensional variables by their characteristic scales. Here, we use the typical orders of magnitude of the physical parameters representative for the Ameland Inlet system, see Table 1.

The relation between the dimensional and dimensionless variables is given by

$$
\begin{array}{ll}
x=L x^{*}, & t=t^{*} \sigma^{-1}, \quad u=U u^{*} \\
\zeta=A_{M_{2}} \zeta^{*}=\frac{H U}{\sigma L} \zeta^{*}, & C=\frac{\alpha U^{2} \kappa_{v}}{\omega_{s}^{2}} C^{*}, \quad h=H h^{*}, W=W_{0} W^{*}
\end{array}
$$

with $W_{0}$ the width at the entrance of the inlet. To relate the velocity scale to the tidal forcing, we use mass

Table 1 Characteristic values for the Ameland Inlet embayment, see Ridderinkhof and Zimmerman (1992), Van Rijn (1993), and Dyer (1986)

\begin{tabular}{ll}
\hline Channel & Sediment \\
\hline$L=20 \cdot 10^{3} \mathrm{~m}$ & $c_{d}=0.001$ \\
$H=12 \mathrm{~m}$ & $g=9.81 \mathrm{~ms}^{-2}$ \\
$B=2 \cdot 10^{3} \mathrm{~m}$ & $\kappa_{h}=10^{2} \mathrm{~m}^{2} \mathrm{~s}^{-1}$ \\
& $\omega_{s}=0.015 \mathrm{~m} \mathrm{~s}^{-1}$ \\
Tide & $\kappa_{v}=0.1 \mathrm{~m}^{2} \mathrm{~s}^{-1}$ \\
$A_{M_{2}}=0.84 \mathrm{~m}$ & $\alpha=0.02 \mathrm{~kg} \mathrm{~s} \mathrm{~m}^{-4}$ \\
$A_{M_{4}}=0.08 \mathrm{~m}$ & $\rho_{s}=2650 \mathrm{~kg} \mathrm{~m}^{-3}$ \\
$\sigma=1.4 \cdot 10^{-4} \mathrm{~s}^{-1}$ & $p=0.4$ \\
$\phi=195^{\circ}$ & $\Gamma=7.8 \cdot 10^{-5}$ \\
$T=44.9 \cdot 10^{3} \mathrm{~s}$ & $u_{c}=0.3 \mathrm{~m} \mathrm{~s}^{-1}$ \\
& $\mu=1.4 \cdot 10^{-4} \mathrm{~m}^{2} \mathrm{~s}^{-1}$ \\
\hline
\end{tabular}

This embayment can be found in the Dutch part of the Wadden Sea. Here the width at the seaward side is chosen as the characteristic width conservation arguments. Requiring the first and the last term of the continuity equation to be of similar order leads to $U \sim \frac{A_{M_{2}} \sigma L}{H}$. Moreover, we require an approximate balance between sediment deposition and erosion. Hence, the scaling of the sediment concentration $C$ is obtained by requiring $\alpha U^{2} \sim \frac{\omega_{s}^{2}}{\kappa_{v}} C$ where we assume $\beta$ to be $\mathcal{O}(1)$.

After substituting the dimensionless variables, the dimensionless model equations are given by

$$
\begin{gathered}
W^{*} \zeta_{t^{*}}^{*}+\left[W^{*}\left(1-h^{*}+\epsilon \zeta^{*}\right) u^{*}\right]_{x^{*}}=0 \\
u_{t^{*}}+\epsilon u^{*} u_{x^{*}}^{*}+\frac{\tilde{r} u^{*}}{1-h^{*}+\epsilon \zeta^{*}}+\Delta^{2} \zeta_{x^{*}}^{*}=0 \\
v W^{*} C_{t^{*}}^{*}+v \epsilon\left(W^{*} C^{*} u^{*}\right)_{x^{*}}-v \kappa\left(W^{*} C_{x^{*}}^{*}\right)_{x^{*}}=W^{*}\left(u^{*^{2}}-\tilde{\beta} C^{*}\right) \\
W^{*} h_{t^{*}}^{*}+\delta \tilde{\mu}\left(W^{*} h_{x^{*}}^{*}\right)_{x^{*}}+\delta\left\langle W^{*}\left(u^{*^{2}}-\tilde{\beta} C^{*}\right)\right\rangle=0
\end{gathered}
$$

The dimensionless deposition parameter reads

$\tilde{\beta}=\left(1-e^{-\lambda\left(\epsilon \zeta^{*}+1-h^{*}\right)}\right)^{-1}$

all dimensionless parameters are defined in Table 2, along with their characteristic values for the Ameland Inlet, (using Table 1).

The scaled boundary conditions at the seaward side, $x=$ 0 , are given by

$$
\begin{aligned}
\zeta^{*} & =\cos \left(t^{*}\right)+\gamma \cos \left(2 t^{*}-\phi\right), \\
\left\langle u^{*^{2}}-\beta C^{*}\right\rangle & =0, \\
\lim _{\kappa \rightarrow 0} \hat{C}^{*}\left(x^{*}, t^{*}, \kappa\right) & =\hat{C}^{*}\left(x^{*}, t^{*}, \kappa=0\right), \\
h^{*} & =0,
\end{aligned}
$$

Table 2 Dimensionless parameters for the Ameland Inlet

$\epsilon=\frac{U}{\sigma L}=\frac{A_{M} 2}{H} \sim 0.07 \quad \frac{\text { tidal excursion length }}{\text { embayment length }}$

$\tilde{r}=\frac{8 c_{d} A_{M_{2}} L}{3 \pi H^{2}} \sim 0.099$

bottom friction parameter

$\Delta^{2}=\frac{g H}{\sigma^{2} L^{2}} \sim 15.015$

$\left(\frac{\text { tidal wave length }}{\text { embayment length }}\right)^{2}$

$v=\frac{\sigma \kappa_{v}}{\omega_{s}^{2}} \sim 0.0622$

$\kappa=\frac{\kappa_{h}}{\sigma L^{2}} \sim 1.79 \cdot 10^{-3}$

$\lambda=\frac{H \omega_{s}}{\kappa_{v}} \sim 1.8$

$\delta=\frac{\alpha U^{2}}{\sigma H \rho_{s}(1-p)} \sim 7.19 \cdot 10^{-5}$

$\gamma=\frac{A_{M_{4}}}{A_{M_{2}}} \sim 0.095$

deposition timescale tidal period

$$
\text { tidal period }
$$

diffusive timescale

vertical diffusion timescale deposition timescale

tidal period morphodynamic timescale

$\frac{M_{4} \text { amplitude }}{M_{2} \text { amplitude }}$

$\tilde{\mu}=\frac{\mu}{\sigma L^{2}} \sim 2.5 \cdot 10^{-9}$ 
where $\gamma$ is the ratio of the $M_{4}$ and the $M_{2}$ tidal amplitude. At the landward side, $x^{*}=\hat{x}$, the scaled boundary conditions result in

$$
\begin{aligned}
u^{*} & =\hat{x}_{t}^{*}, \\
-v \kappa\left\langle W^{*} C_{x^{*}}^{*}\right) & =0, \\
\lim _{\kappa \rightarrow 0} \hat{C}^{*}\left(x^{*}, t^{*}, \kappa\right) & =\hat{C}^{*}\left(x^{*}, t^{*}, \kappa=0\right) .
\end{aligned}
$$

We use the approach taken in Ter Brake and Schuttelaars (2010) to transform the moving boundary $x^{*}=\hat{x}$ into a fixed boundary condition at $x^{*}=1$. The moving boundary, $\hat{x}$, can be determined by studying the intersection point of the bed and the water level which is given by the following relation

$1-h^{*}\left(x^{*}\right)+\epsilon \zeta^{*}\left(x^{*}, t^{*}\right)=0$.

Next we use that the tidally averaged condition implies that the length of the scaled embayment is 1 . After substituting this condition into the continuity equation, we reformulate the boundary conditions at the landward side. Since the boundary is now fixed, there is sediment transport due to both diffusion and advection at the boundary. Therefore, at $x^{*}=1$, the boundary conditions read

$$
\begin{aligned}
& u_{x^{*} \text { is finite }}, \\
& <\epsilon\left(W^{*} C^{*} u^{*}\right)-\kappa\left(W^{*} C_{x^{*}}^{*}\right)>=0, \\
& \lim _{\kappa \rightarrow 0} \hat{C}^{*}\left(x^{*}, t^{*}, \kappa\right)=\hat{C}^{*}\left(x^{*}, t^{*}, \kappa=0\right)
\end{aligned}
$$

Using Table 2, we observe that the parameter $\delta \sim$ $7.2 \cdot 10^{-5}$ is small. Therefore, it follows from Eq. 3.5 that $h_{t^{*}}^{*}=\mathcal{O}(\delta)$ and hence the bed level is constant on the $\mathcal{O}(1)$-timescale, which is the fast hydrodynamic timescale. Therefore, a new slow time coordinate is introduced, $\tau^{*}=$ $\delta t^{*}$ on which $h^{*}$ does vary. Upon tidally averaging Eq. 3.4, we obtain $\left\langle v \epsilon\left(W^{*} C^{*} u^{*}\right)_{x^{*}}-v \kappa\left(W^{*} C_{x^{*}}^{*}\right)_{x^{*}}\right\rangle=\left\langle W\left(u^{*^{2}}-\right.\right.$ $\left.\left.\tilde{\beta} C^{*}\right)\right\rangle$. Substituting this expression for the tidally averaged erosion and deposition flux into the bed evolution Eq. 3.5, we obtain, written in terms of the slow timescale,

$W h_{\tau}^{*}=-<F^{*}>_{x^{*}}$.

Here, the dimensionless sediment transport $F^{*}$ consists of bed slope effects of bedload transport and diffusive and advective contributions of the suspended sediment transport,

$F^{*}=F_{\mathrm{bl}}^{*}+F_{\mathrm{diff}}^{*}+F_{\mathrm{adv}}^{*}$,

with

$$
\begin{aligned}
F_{\mathrm{bl}}^{*} & =-\tilde{\mu} W^{*} h_{x^{*}}^{*}, \\
F_{\mathrm{diff}}^{*} & =-v \kappa W^{*} C_{x^{*}}^{*}, \\
F_{\mathrm{adv}}^{*} & =v \epsilon W^{*} u^{*} C^{*} .
\end{aligned}
$$

The boundary condition at the landward side for the residual sediment concentration can also be given in terms of these sediment transport and reads

$$
<F_{\mathrm{bl}}^{*}+F_{\mathrm{diff}}^{*}+F_{\mathrm{adv}}^{*}>=0 .
$$

\subsection{The analysis of the model}

For some simplified situations, the model can be analytically solved, see Schuttelaars and de Swart (1996). However, in general the model has to be solved numerically. As indicated above, the bed evolves due to convergences of different types of sediment transport. In this article, we study the influence of the contribution of these different processes. To systematically assess the importance of the various transport contributions, the model output is analysed in terms of these transport contributions. Leaving out the slope contribution of the bedload transport is not allowed as this would make it impossible to impose boundary conditions for the bed evolution equation at the seaward and landward side. Since it is essential to impose these boundary conditions, at least the slope term of the bedload component has to be retained, although its contribution is (at least in the main part of the embayment) negligible.

We consider two types of advective sediment transport. We define external advective transport as transport due to those advective processes that occur when an externally prescribed overtide is included and internal advective transport as transport due to those advective processes that occur due to the nonlinear interactions inside the embayment. Note that both temporal and spatial settling lag effects (Burchard et al. 2018) contribute to the external and internal advective transport. The sediment transport due to the bed slope effect is a diffusive term in the bed level equation. In this analysis, we make use of the values of Table 2, which indicates that both $\epsilon \ll 1$ and $\gamma \ll 1$. Since $\gamma$ is associated with the external advective processes and $\epsilon$ with the internal advective processes, we treat these two parameters as independent to distinguish between the two advective processes instead of introducing one small parameter. Note that these parameters are not necessarily of different order. We expand all variables in terms of the small parameters as

$X=X^{0,0}+\epsilon X^{1,0}+\gamma X^{0,1}+$ h.o.t.,

where $X=\{\zeta, u, C\}$. The first superscript denotes the order of the variable in $\epsilon$ and the second the order in $\gamma$.

We determine which terms of the sediment transport $F^{*}$ that are present in Eq. 3.7 are time-independent since only these terms contribute to the bed evolution. To do this, we decompose all terms in expression (3.9) in their fluctuating 
parts and their residual component as follows

$X^{k, j}=<\chi^{k, j}(x)>+\sum_{\omega} \mathcal{R}\left(\chi_{\omega}^{k, j}(x) e^{-i \omega t}\right)$,

where the frequency of the time-dependent components is denoted by $\omega$ and $\mathcal{R}$ denotes taking the real part of the expression. Here, $\chi^{k, j}(x)$ and $\chi_{\omega}^{k, j}(x)$ denote the spatial dependency of the residual and the fluctuating parts of the solution, respectively. Note that, in general, $\chi_{\omega}^{k, j}(x)$ is a complex function. We only consider the $M_{0}$ (residual component), $M_{2}$ and $M_{4}$ tidal constituents, and assume that all other tidal constituents are much smaller and can be neglected. Using the boundary conditions for the sea surface elevation and the nonlinear terms in the sediment transport equation, we can deduce which tidal constituent contributes to the velocity, the sea surface elevation and the sediment concentration at different orders, see Table 3.

We substitute expansion (3.9) into Eqs. 3.2-3.4 and collect terms of equal order to obtain model equations at leading order, $\mathcal{O}(\epsilon)$ and $\mathcal{O}(\gamma)$. We rewrite the equations at the different orders back into their dimensional form by using the transformations (3.1) and the expressions in Table 2. This way, we can immediately study the influence of the physical parameters on solutions to the model. At leading order, the dimensional equations read

$$
\begin{aligned}
& W \zeta_{t}^{0,0}+\left[W(H-h) u^{0,0}\right]_{x}=0 \\
& u_{t}^{0,0}+\frac{r u^{0,0}}{H-h+h_{0}}+g \zeta_{x}^{0,0}=0 \\
& W C_{t}^{0,0}-\kappa_{h}\left(W C_{x}^{0,0}\right)_{x}-W\left(\alpha u^{0,0^{2}}-\frac{\omega_{s}^{2}}{\kappa_{v}} \beta^{0,0} C^{0,0}\right)=0
\end{aligned}
$$

Since the deposition parameter $\beta$, see Eq. 3.6, also depends on $\epsilon$, only the leading order contribution,

$\beta^{0,0}=\left[1-h_{\delta} e^{-\frac{\omega_{s}}{\kappa_{v}}(H-h)}\right]^{-1}$,

is used in the equation above. We have introduced constants $0<h_{\delta}<1$ and $h_{0} \ll 1$ to prevent divergences of the deposition and bottom friction term, respectively. From the scaling arguments, we should conclude that the diffusion term is much smaller than the other terms, $(\nu \kappa \sim$ $\mathcal{O}\left(10^{-4}\right)$ ), and therefore, should not be present in the leading order sediment concentration equation. However, a residual concentration term can lead to a diffusive boundary

Table 3 The tidal constituents which contribute to the velocity, the sea surface elevation and the sediment concentration for the leading (order one), $\epsilon$ and $\gamma$ orders

\begin{tabular}{llll}
\hline & $\mathcal{O}(1)$ & $\mathcal{O}(\epsilon)$ & $\mathcal{O}(\gamma)$ \\
\hline$u, \zeta$ & $M_{2}$ & $M_{0}, M_{4}$ & $M_{4}$ \\
$C$ & $M_{0}, M_{4}$ & $M_{2}$ & $M_{2}$ \\
\hline
\end{tabular}

layer. This is a thin layer at the boundaries where the diffusive transport can still be significant, compared to the other transport contributions. For that reason, the diffusion term has to be taken into account at leading order. The leading order boundary conditions are given by

$$
\begin{aligned}
\zeta^{0,0}=A_{M_{2}} \cos (\sigma t) & \text { at } x=0, \\
W(H-h) u^{0,0}=0 & \text { at } x=L, \\
<\alpha u^{0,0^{2}}-\frac{\omega_{s}^{2}}{\kappa_{v}} \beta C^{0,0}>=0 & \text { at } x=0 \\
\lim _{\kappa_{h} \rightarrow 0} \hat{C}^{0,0}\left(x, t, \kappa_{h}\right)=\hat{C}^{0,0}\left(x, t, \kappa_{h}=0\right) & \text { at } x=0, L .
\end{aligned}
$$

At $\mathcal{O}(\gamma)$, there is no residual concentration. Therefore, we do not need the diffusion term to compensate for a potential diffusive boundary layer. Hence, the system of equations at $\mathcal{O}(\gamma)$ reads:

$$
\begin{aligned}
W \zeta_{t}^{0,1}+\left[W(H-h) u^{0,1}\right]_{x} & =0, \\
u_{t}^{0,1}+\frac{r u^{0,1}}{H-h+h_{0}}+g \zeta_{x}^{0,1} & =0, \\
W C_{t}^{0,1}-W\left(2 \alpha u^{0,0} u^{0,1}-\frac{\omega_{s}^{2}}{\kappa_{v}} \beta^{0,0} C^{0,1}\right) & =0,
\end{aligned}
$$

with corresponding boundary conditions

$$
\begin{aligned}
& \zeta^{0,1}=A_{M_{4}} \cos (2 \sigma t-\phi) \quad \text { at } \quad x=0, \\
& W(H-h) u^{0,1}=0 \quad \text { at } \quad x=L, \\
& \lim _{\kappa_{h} \rightarrow 0} \hat{C}^{0,1}\left(x, t, \kappa_{h}\right)=\hat{C}^{0,1}\left(x, t, \kappa_{h}=0\right) \quad \text { at } \quad x=0, L \text {. }
\end{aligned}
$$

At $\mathcal{O}(\epsilon)$, again neglecting the diffusive sediment transport, the equations read

$$
\begin{aligned}
W \zeta_{t}^{1,0} & +\left[W(H-h) u^{1,0}\right]_{x}+\left[W \zeta^{0,0} u^{0,0}\right]_{x}=0 \\
u_{t}^{1,0} & +u^{0,0} u_{x}^{0,0}+\frac{r u^{1,0}}{H-h+h_{0}}+g \zeta_{x}^{1,0}+\frac{r u^{0,0} \zeta^{0,0}}{\left(H-h+h_{0}\right)^{2}}=0 \\
W C_{t}^{1,0} & +\left(W C^{0,0} u^{0,0}\right)_{x} \\
& -W\left(2 \alpha u^{0,0} u^{1,0}-\frac{\omega_{s}^{2}}{\kappa_{v}} \beta^{0,0} C^{1,0}-\frac{\omega_{s}^{2}}{\kappa_{v}} \beta^{1,0} C^{0,0}\right)=0 .
\end{aligned}
$$

Here, the $\mathcal{O}(\epsilon)$-term in the deposition parameter $\beta$, Eq. 3.6 has to be taken into account. It is given by

$\beta^{1,0}=\frac{\omega_{s} h_{\delta}}{\kappa_{v}} e^{-\frac{\omega_{s}}{\kappa_{v}}(H-h)}\left(1-h_{\delta} e^{-\frac{\omega_{s}}{\kappa_{v}}(H-h)}\right)^{-2}$.

The corresponding boundary conditions are given by

$$
\begin{aligned}
& \zeta^{1,0}=0 \quad \text { at } \quad x=0, \\
& W(H-h) u^{1,0}+W \zeta^{0,0} u^{0,0}=0 \quad \text { at } \quad x=0, \\
& \lim _{\kappa_{h} \rightarrow 0} \hat{C}^{1,0}\left(x, t, \kappa_{h}\right)=\hat{C}^{1,0}\left(x, t, \kappa_{h}=0\right) \quad \text { at } \quad x=0, L \text {. }
\end{aligned}
$$




\subsection{Morphodynamic equilibria}

Next, we study the existence of morphodynamic equilibria. We want to emphasise that we do not use time-integration to obtain morphodynamic equilibria. This implies that we look for a morphodynamic equilibrium, given the length of the inlet system and a fixed depth at the entrance. We make use of the fact that a morphodynamic equilibrium arises when $h_{\tau}=0$, and hence, using Eq. 3.7 and boundary condition (3.8) we find that

$<F>=0$,

with the dimensional sediment transport $F$ given below by expression (3.14). This means that the sum of the tidally averaged diffusive and advective suspended sediment transports and the bed slope effects of the bedload transport have to balance at each location in the embayment. To obtain these tidally averaged transports, we use the tidal constituent information of the velocity $u$ and the sediment concentration $C$, given in Table 3, and the expressions for the sediment transport (3.14). All terms with periodic behaviour average to zero after tidal averaging. Therefore, only the terms of the sediment transport which are time-independent lead to a contribution. This reveals that the leading order tidally averaged internal advective sediment transport is $\mathcal{O}\left(v \epsilon^{2}\right)$, the external advective sediment transport is $\mathcal{O}(v \in \gamma)$ and the diffusive sediment transport is $\mathcal{O}(\nu \kappa)$. Using the parameter values given in Table 1, this means that all types of sediment transport are of the same order, and hence, have to be taken into account. In terms of the regular expansion, the total tidally averaged sediment transport consists to leading order of four components

$<F>=<F_{\mathrm{bl}}^{0,0}>+<F_{\text {diff }}^{0,0}>+<F_{\mathrm{adv}}^{2,0}>+<F_{\mathrm{adv}}^{1,1}>$,

with their dimensional expressions given by

$$
\begin{aligned}
& <F_{\mathrm{bl}}^{0,0}>=-\rho_{s}(1-p) \mu W h_{x}, \\
& <F_{\text {diff }}^{0,0}=-\kappa_{h}<W C_{x}^{0,0}>, \\
& <F_{\text {adv }}^{2,0}>=<W\left(u^{0,0} C^{1,0}+u^{1,0} C^{0,0}\right)>, \\
& <F_{\text {adv }}^{1,1}>=<W\left(u^{0,0} C^{0,1}+u^{0,1} C^{0,0}\right)>.
\end{aligned}
$$

We denote the transport due to internal advection by $F^{2,0}$ and due to external advection by $F^{1,1}$.

\subsection{Numerical method}

To obtain morphodynamic equilibria, the model equations at leading order, order $\epsilon$ and $\gamma$ are solved numerically using a finite element method. As a first step, we convert the model equations into their weak formulation and we discretise the model equations using piecewise linear functions. We apply a so-called continuation method: starting with a known equilibrium profile for a specific set of parameters as an initial guess, one can obtain equilibria by slowly varying parameters or the geometry using a NewtonRaphson procedure. The morphodynamic equilibrium that we use as a starting point in the continuation method is the morphodynamic equilibrium of a short, rectangular embayment where sediment transport is dominated by diffusive transport and inertia and friction are neglected. This equilibrium bed profile is constantly sloping, see Schuttelaars and de Swart $(1996,2000)$ and Ter Brake and Schuttelaars (2010).

\section{Results}

Single tidal inlet systems consist of a back barrier basin and one inlet connecting the basin to the sea. Typically, single tidal embayments in the Wadden Sea have an embayment length between $10 \mathrm{~km}$ and $20 \mathrm{~km}$. Hence, we focus on analysing the morphodynamic equilibria in embayments with lengths between $L=8 \mathrm{~km}$ and $L=$ $22 \mathrm{~km}$. Although we allow for arbitrary width variations, to systematically analyse the effect of width variations on the morphodynamic equilibria, we mainly focus on exponentially converging and diverging inlets:

$$
W(x)=W_{0} e^{-\frac{x}{L_{b}}} \text {. }
$$

Here, $W_{0}$ the width of the embayment at the entrance and $L_{b}$ the exponential convergence length. Our first results show the influence of geometrical variations on the morphodynamic equilibria by keeping the length $L=$ $20 \mathrm{~km}$ fixed and varying the convergence length. Next, we vary the length of the system by keeping $W_{0}=2 \mathrm{~km}$ and $L_{b}$ constant. This results in a varying width at the landward side. We consider three different geometries with profiles sketched in Fig. 1: an exponentially converging, rectangular and exponentially diverging embayment. Unless stated otherwise, we use the characteristic values of the Ameland Inlet system, given in Table 1.

\subsection{Diffusively dominated transport}

In this section, we assume that the suspended load transport is dominated by diffusive processes.

Geometry We start by studying the effect of variations in the geometry of the embayment on morphodynamic equilibria. Here, we neglect frictional effects in the water motion $\left(c_{d}=0\right)$ and we assume that the deposition parameter is depth-independent $(\beta=1)$. In Fig. 2, the resulting equilibrium bed profiles are shown for an 


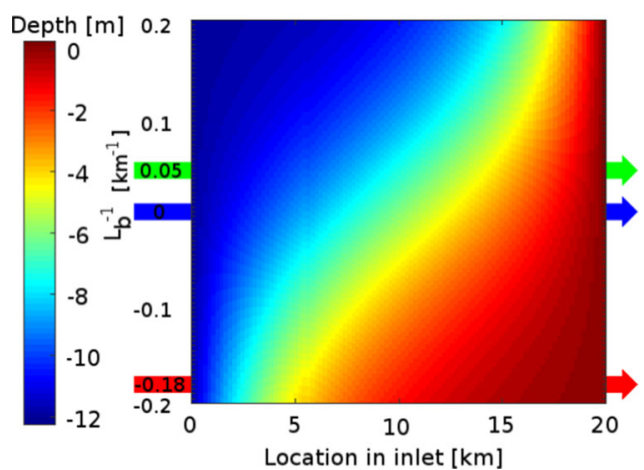

(a)

Fig. 2 The influence of geometrical variations on the morphodynamic equilibria. a Equilibrium bed profiles are shown as a function of the location in the tidal embayment (horizontal axis) for inlets that range from strongly converging, $L_{b}^{-1}=0.2 \mathrm{~km}^{-1}$, to strongly diverging, $L_{b}^{-1}=-0.2 \mathrm{~km}^{-1}$ (vertical axis). Using the default embayment width at the seaward side of $W_{0}=2 \mathrm{~km}, \frac{L}{L_{b}}=1$ corresponds to a landward width of $0.7 \mathrm{~km}$ and $\frac{L}{L_{b}}=-1$ to a landward width of $5.4 \mathrm{~km}$.

embayment with a length of $L=20 \mathrm{~km}$ for different convergence lengths.

For the rectangular inlet (blue line in Fig. 2b), we observe that the water depth constantly decreases. This result is similar to what was already found in Schuttelaars and de Swart (1996) and Ter Brake and Schuttelaars (2010), using a model formulation in which inertia was assumed to be negligible in the momentum equation. Our results show that inertia does not influence the bed equilibrium profile. For a tidal embayment with width variations in the along-channel direction, it is found that for a more diverging (converging) inlet, a more convex (concave) equilibrium bed profile is obtained.

To explain this dependency, we neglect the bedload transport. Then, the morphodynamic equilibrium condition is attained when $F_{\text {diff }}^{0,0}=-\kappa_{h}<W C_{x}^{0,0}>=0$, in other words, $<C^{0,0}>$ must be constant in morphodynamic equilibrium. Assuming that erosion and deposition balance approximately, it follows from Eq. 3.12 that

$<C^{0,0}>\approx<u^{0,0^{2}}>$.

Hence, in morphodynamic equilibrium the amplitude $u^{0,0}$ must be independent of $x$ since $C^{0,0}$ has to be independent of $x$. This result is consistent with findings in Friedrichs and Aubrey (1996), Pritchard and Hogg (2003), Toffolon and Lanzoni (2010), and Todeschini et al. (2008). Using the boundary conditions at the seaward boundary and that local inertia is negligible, the momentum Eq. 3.11 implies that the sea surface elevation behaves like $\zeta^{0,0} \approx A_{M_{2}} \cos (\sigma t)$.

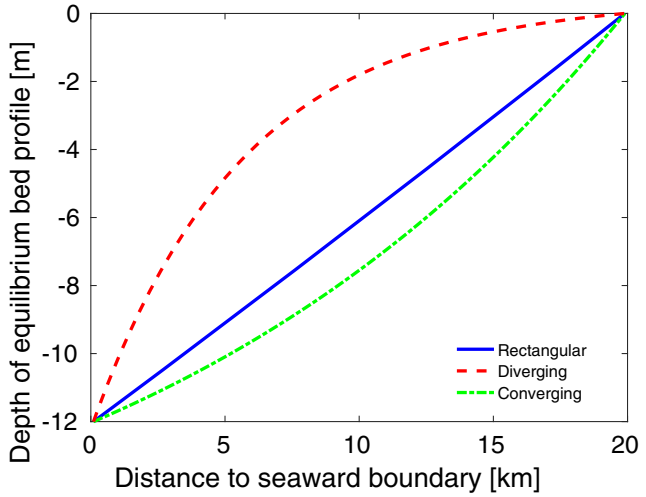

(b)

The water depth is indicated by the colour coding where the colder (warmer) colours correspond to larger (smaller) dephts. b Equilibrium bed profiles are shown for a rectangular embayment (infinite convergence length $L_{b}$, solid blue line), a diverging embayment ( $L_{b} \approx$ $-5.6 \mathrm{~km}$, dashed red line $)$ and a converging embayment $\left(L_{b}=20 \mathrm{~km}\right.$, dashed-dotted green line)

Then, from Eq. 3.10, it follows that the resulting velocity becomes

$u^{0,0} \approx \frac{\sigma A_{M_{2}} L_{b}}{H-h}\left(1-e^{\frac{x-L}{L_{b}}}\right) \sin (\sigma t)$.

Now, requiring that the amplitude of $u^{0,0}$ does not depend on $x$, we find that

$h(x) \sim H-\sigma A_{M_{2}} L_{b}\left(1-e^{\frac{x-L}{L_{b}}}\right)$.

From this, we conclude that for a converging embayment $\left(L_{b}>0\right)$, the first and second derivative of the depth are positive and hence, the morphodynamic profile is concave. On the other hand, for a diverging embayment $\left(L_{b}<0\right)$, the first derivative is still positive, but the second derivative is negative, resulting in a convex bed profile.

Deposition parameter In the previous paragraph, morphodynamic equilibria were obtained assuming a constant deposition parameter, $\beta^{0,0}=1$, i.e. $h_{\delta}=0$. Here, the influence of a depth-dependent deposition parameter on the resulting equilibria is investigated by comparing the results obtained with $h_{\delta}=0$ (no depth-dependent deposition parameter) and $h_{\delta}=0.9$ (depth-dependent deposition parameter). Note that we do not take $h_{\delta}=1.0$ to avoid singularities. To highlight the influence of depth-dependent deposition on the morphodynamic equilibria, the bed profiles are subtracted from each other as shown in the left column of Fig. 3 for various embayment geometries.

We observe that for a rectangular inlet, the equilibrium bed profile becomes convex when the depth-dependency of the deposition parameter is incorporated. This result 
Fig. 3 The influence of the deposition parameter for diverging (top), rectangular (middle) and converging (bottom) embayment geometries. In the colourplots in the left column, the difference of the bed profiles

$\left(\operatorname{sol}_{h_{\delta}=0.9}-\operatorname{sol}_{h_{\delta}=0}\right)$ is shown as a function of the distance to the seaward boundary (horizontal axis) and the embayment length (vertical axis). The colour indicates the effect of the deposition parameter on the morphodynamic equilibria: blue colours indicate that the local water depth is not sensitive to the depth-dependence of the deposition parameter, warmer colours indicate that the difference between the local water depth with and without depth-dependent deposition increases. In the column on the right, examples of equilibrium bed profiles are shown for different basin lengths with (dashed) and without (solid) a depth-dependent deposition parameter
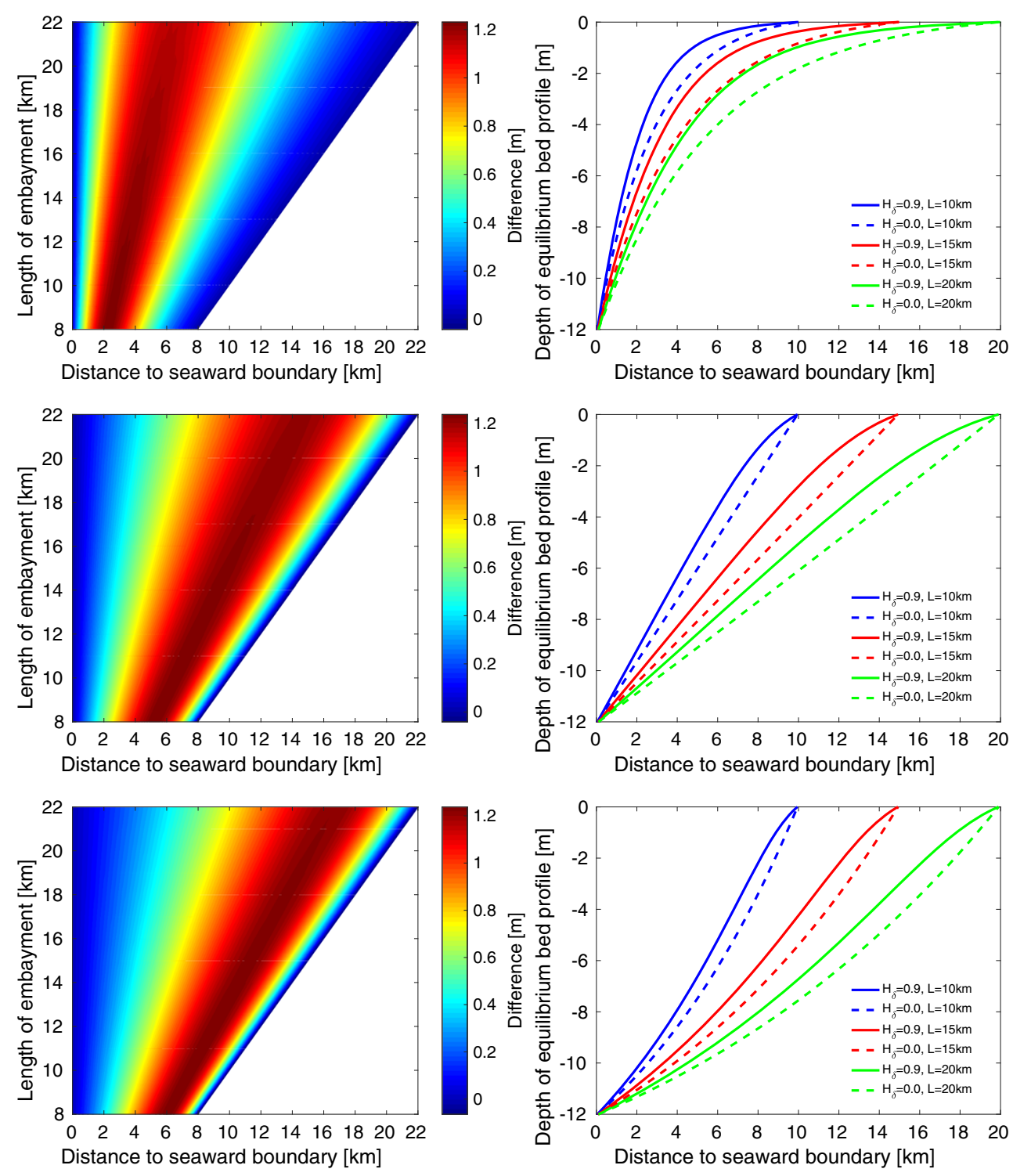

is in agreement with earlier findings in Ter Brake and Schuttelaars (2010) and Van Leeuwen et al. (2000). We see that the same change also occurs when the geometry of the embayment is varied in the along-channel direction. The location where the solution is most convex is found in the middle of the embayment for a diverging geometry and lies more towards the landward boundary as the embayment becomes more converging.

These results can be explained by considering the morphodynamic equilibrium condition, again neglecting the bedload transport. We find that in order for $F_{\text {diff }}^{0,0}=-\kappa_{h}<$ $W C_{x}^{0,0}>=0$ to be satisfied, the leading order residual sediment concentration, $C^{0,0}$ cannot depend on $x$. Using this in Eq. 3.12, it follows that $<\beta^{0,0} C^{0,0}>\approx<u^{0,0^{2}}>$. Since $C^{0,0}$ has to be spatially uniform, this implies that $\frac{\left(u^{0,0}\right)^{2}}{\beta^{0,0}}$ must also be spatially uniform, where $\beta^{0,0}=$ $\left[1-h_{\delta} e^{-\frac{\omega_{s}}{\kappa_{v}}(H-h)}\right]^{-1}$. From Fig. 3, we deduce that the bed profiles are non-decreasing functions of the longitudinal coordinate $x$. Since the exponent of the leading order deposition parameter depends on the bed profile, this implies that the leading order deposition parameter increases towards the landward boundary when $h_{\delta}$ is non-zero. Therefore, for $\frac{\left(u^{0,0}\right)^{2}}{\beta^{0,0}}$ to remain spatially uniform, the amplitude $u^{0,0}$ of the velocity has to increase towards the landward boundary as well. Using expression (4.3), we deduce that for the velocity to increase, $H-h$ has to decrease, i.e. the water depth has to decrease.

Friction To study the influence of the bottom friction on the morphodynamic equilibria, we take the deposition parameter to depend on the depth, $h_{\delta}=0.9$, and add frictional effects by setting $c_{d}=0.001$ and $c_{d}=0.003$. 
Again, we vary the length of the tidal embayment and subtract the solutions obtained with and without friction. The results for a diverging embayment are shown in Fig. 4. The figure shows that bottom friction becomes more important for longer embayments. Including frictional effects leads to more convex bed profiles, although the concave-profile of the bed equilibrium for a converging geometry is still present. This latter result agrees with findings of Lanzoni and Seminara (2002). The influence for a converging and rectangular embayment is qualitatively the same, although the influence is much smaller.

The above results can be explained as follows. The bottom friction parameter depends linearly on the embayment length $L$. This implies that as the length of the embayment increases, the bottom friction term becomes more important. Furthermore, the bottom friction term is divided by the local water depth in the leading order momentum equation, (3.11). This suggests that this term becomes more significant when the local water depth decreases. As we have seen in the previous section when friction was neglected, the bed is much shallower for a diverging embayment than for a converging one. Therefore, if friction effects are added, their influence is largest for a diverging inlet.

\subsection{Advective and diffusive transport without an externally prescribed overtide}

In this section, we analyse the influence of advective sediment transport due to internally generated overtides and the associated suspended sediment concentrations on the morphodynamic equilibria. The deposition parameter is taken to be depth-dependent and frictional effects are included. Again, the solutions with internal advection and without (from Section 4.1) are subtracted from each other. In Fig. 5, the results are shown for various geometries.

For a rectangular inlet, we find that the morphodynamic bed equilibrium is less convex compared to the case where only diffusion is considered. This result is similar to the findings of Ter Brake and Schuttelaars (2010), although the effect of adding internal advection is stronger in our study which is due to frictional effects. When only diffusion is considered, friction only resulted in minor adjustments to the equilibrium bed profile, but the influence of friction is not negligible anymore when internal advection is considered. From Fig. 5, we conclude that for a larger embayment length, the internal advective processes become more important. Also, the importance of internally generated advective sediment transport becomes larger for more strongly divergent tidal inlets widths, for which the equilibrium bed profile is very shallow towards the landward side of the basin. Furthermore, when including internal advection the velocities decrease (increase) into the landward direction when the system is diverging (converging).

To explain this influence, we again study the morphodynamic equilibrium condition, neglecting bedload transport, which now reads,

$-\kappa_{h} W<C_{x}^{0,0}>+W<u^{0,0} C^{1,0}+u^{1,0} C^{0,0}>=0$.

For a morphodynamic equilibrium when only diffusive processes are taken into account, the first term of Eq. 4.4 is zero whereas the other terms are negative. This implies that when advection is included, the advective sediment transport due to internal processes is directed towards the sea. Therefore, for this equilibrium condition to hold, the diffusive sediment transport needs to become positive. From

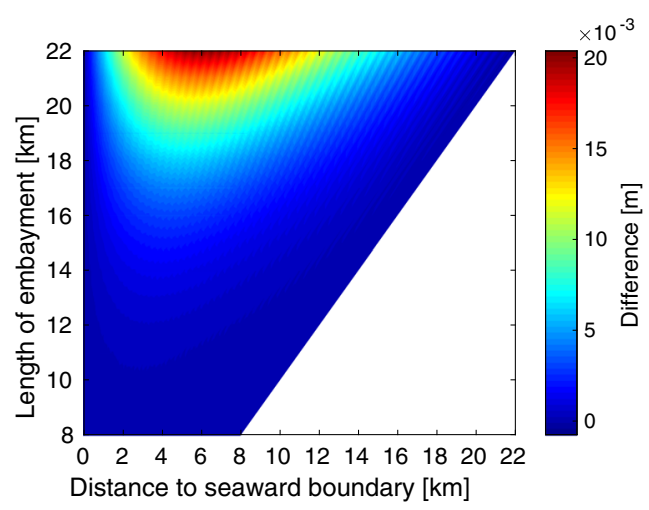

Fig. 4 The influence of the bottom friction parameter for a diverging embayment. The colourplots show the difference between the bed profiles when friction is included and not included, left: $\left(\mathrm{sol}_{c_{d}}=0.001-\right.$ $\left.\operatorname{sol}_{C_{d}=0}\right)$ and right: $\left(\operatorname{sol}_{c_{d}=0.003}-\operatorname{sol}_{C_{d}=0}\right)$. The embayment length is varied between $L=8 \mathrm{~km}$ and $L=22 \mathrm{~km}$ (vertical axis) and the distance to the seaward boundary on the horizontal axis. The colour indicates the influence of bottom friction: the blue colours indicate that

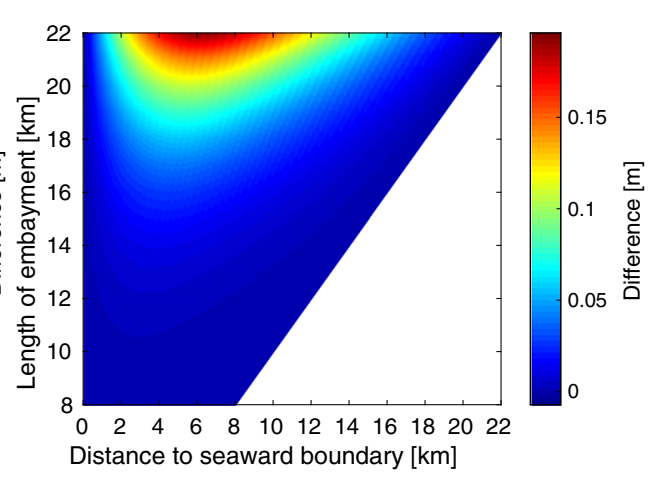

the morphodynamic equilibrium is not sensitive to frictional effects. For warmer colours the influence of bottom friction on the local water depth increases. The figure shows that frictional effects are negligible when the embayment length is $L=8 \mathrm{~km}$ and these effects become stronger as the length of the embayment increases with a maximum difference of $\sim 0.02 \mathrm{~m}$ for $c_{d}=0.001$ and $\sim 0.1 \mathrm{~m}$ for $c_{d}=0.003$ when the embayment length is $L=20 \mathrm{~km}$ 
Fig. 5 The influence of internal advection. The embayment geometry is diverging (top), rectangular (middle) and converging (bottom). a Colourplots of the difference $\left(\mathrm{sol}_{\text {diff }+ \text { adv }}-\mathrm{sol}_{\text {diff }}\right)$ bed profiles are shown for the embayment length versus the distance to the seaward boundary. The colour indicates the effect of including the internal advection on the morphodynamic equilibria: red colours indicate that the local water depth is not sensitive to internal advection, for colours towards the blue the difference between the local water depth with and without internal advective sediment transport increases. b Examples of equilibrium bed profiles are shown for different parameter settings
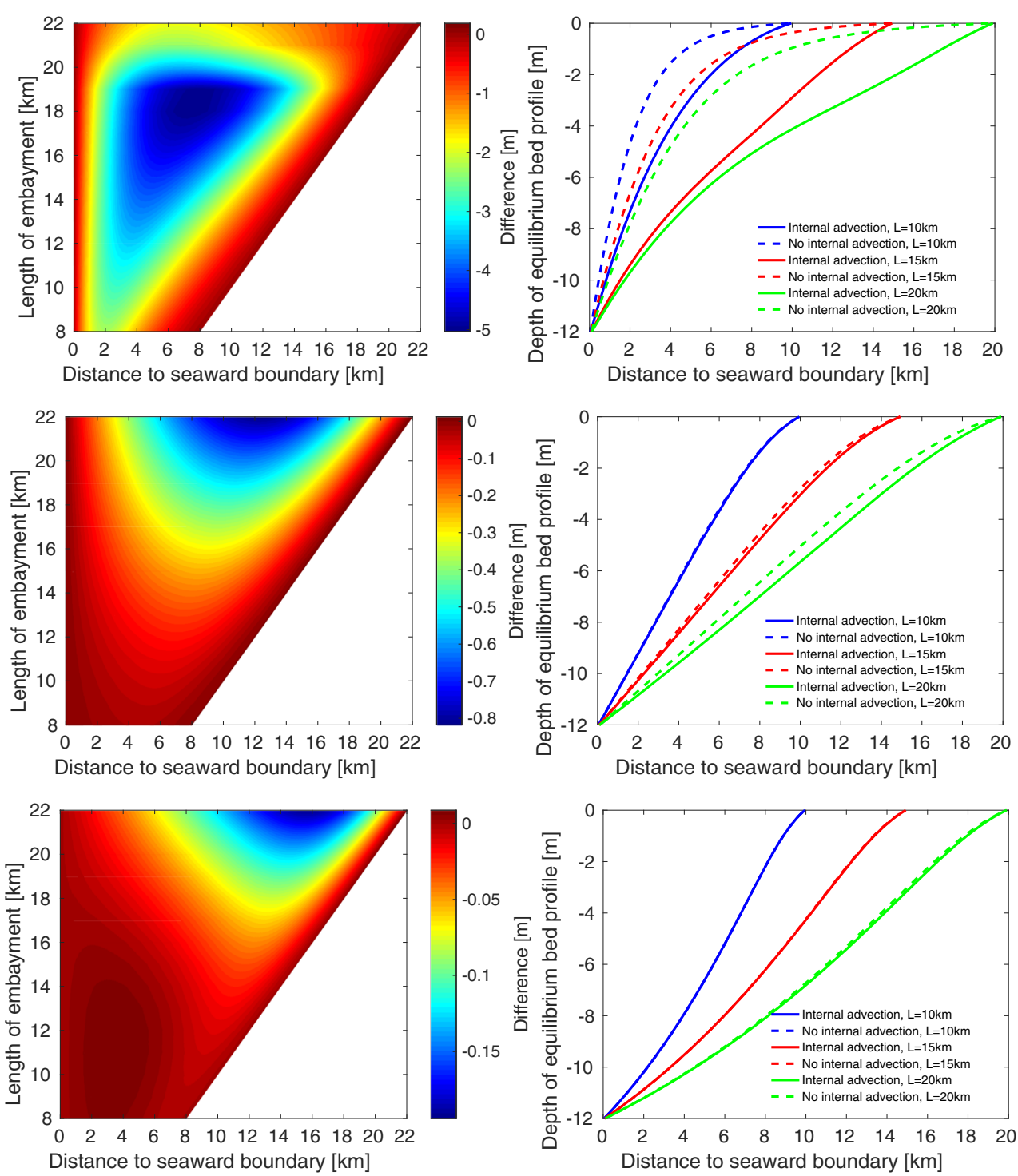

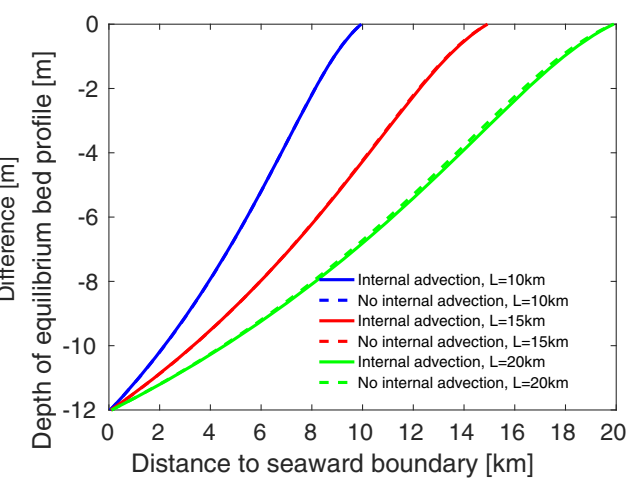

Eqs. 4.3 and 4.2 it follows that this can be achieved by increasing the water depth.

\subsection{Advective and diffusive transport with an externally prescribed overtide}

In this section, the sea surface elevation is forced by both a $M_{2}$ tide and a $M_{4}$ tide. The deposition parameter $\beta$ depends on the depth and frictional effects are taken into account. In discussing the results, we focus on the sensitivity of morphodynamic equilibria to the relative phase of the overtide. We discuss the sensitivity of the maximal tidal embayment length to the relative phase, see for example Schuttelaars and de Swart (2000), Ter Brake and Schuttelaars (2010), Todeschini et al. (2008), and Seminara et al. (2010). To determine the maximum embayment length, morphodynamic equilibria are obtained for each relative phase by increasing the length from $L=$ $8 \mathrm{~km}$. The maximum embayment length is the largest $L$ for which a morphodynamic equilibrium can still be found numerically for the parameters under consideration. If the embayment length $L=22 \mathrm{~km}$ is reached, a maximum length might still exist but it is not in the range of lengths we are focusing on.

\subsubsection{Converging tidal embayment}

In Fig. 6, we give the maximum embayment length as a function of the relative phase. The figure shows that the maximum embayment length significantly changes when varying this parameter, the drag coefficient and the amplitude of the external overtide. For the default parameter values, the smallest maximum length is obtained for $\phi=270^{\circ}$ and is around $L=8 \mathrm{~km}$. We see that 


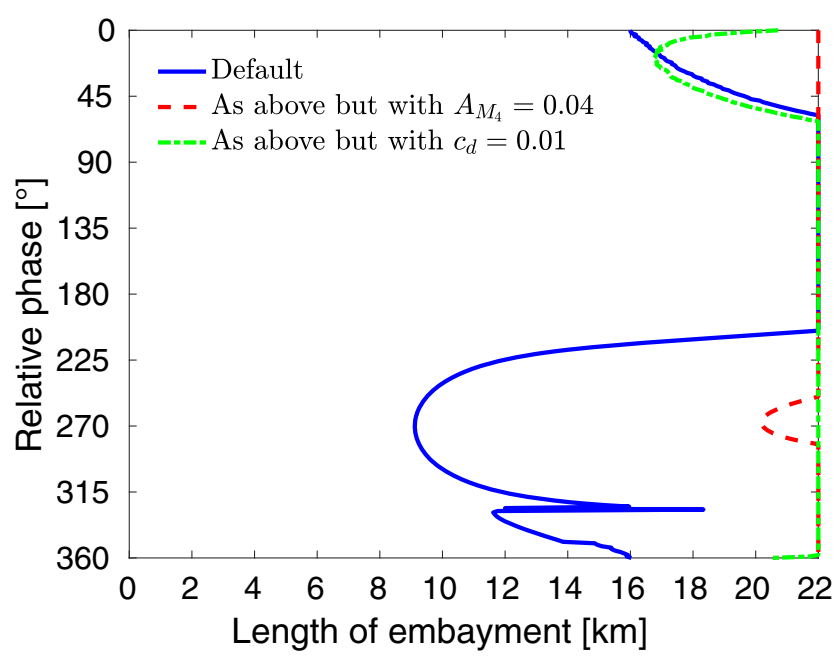

Fig. 6 The relative phase is plotted versus the maximum embayment length (or $22 \mathrm{~km}$ ) for a converging embayment geometry. The blue solid line corresponds to the default parameter values given in Table 1. The other lines are obtained by varying one parameter: $A_{M_{4}}=0.04$ (red dashed line) and $c_{d}=0.01$ (green dashed-dotted line)

when frictional effects are increased, the maximum length increases. Decreasing the amplitude of the $M_{4}$-tide also increases the maximum embayment length.

These results can be explained by analysing the different sediment transport contributions. Decreasing the amplitude of the $M_{4}$ tide results in a smaller contribution of the advective transport due to externally generated overtides. Therefore, it is easier to balance this contribution by the internally generated and diffusive sediment transport, resulting in an increase of the maximum length for all values of the relative phase. Increasing the drag coefficient leads to an increase of the internal advection, resulting in a balance for longer tidal inlet systems.

Even though we observe that the maximum length of the embayment is highly influenced by parameters, we find that the morphodynamic bed equilibria and the balance of their corresponding sediment transport are quite similar when considering the same relative phase. However, when the relative phase is varied, the morphodynamic equilibria change significantly. In Fig. 7 , bed profiles of the morphodynamic equilibria and their corresponding sediment transport are shown for $\phi=195^{\circ}$ and $\phi=67^{\circ}$. We see that for the first choice of $\phi$, the main balance is between the internal and external advective sediment transport, whereas the balance in the latter case is between diffusion and external advection. Note that the net sediment transport remains zero, but the sediment transport due to external advection changes from importing for $\phi=195^{\circ}$ to exporting for $\phi=67^{\circ}$, which agrees with the embayment going from flood dominated to ebb dominated. Also, the bed profile changes from a shallow to a deep one.

\subsubsection{Rectangular tidal embayment}

We performed a similar analysis for a rectangular geometry of the embayment and found that the bed profiles are, apart from being less concave, qualitative the same as those for the converging geometry embayment. The dependency of the maximum length on the relative phase is similar to the relation found for the converging embayment. The maximum length decreases as the convergence length increases for all values of the relative phase which agrees to findings of Seminara et al. (2010). The maximum length for a rectangular embayment forced by an overtide with a relative phase $\phi$ around $270^{\circ}$ is still approximately $L_{\max }=$ $8 \mathrm{~km}$ which was also found by Ter Brake and Schuttelaars (2010).

\subsubsection{Diverging tidal embayment}

When we choose the geometry embayment to be diverging, the values of the relative phase for which there is a maximum length start to differ significantly: there are less parameter combinations for which no morphodynamic equilibrium can be found. The region around $\phi=270^{\circ}$
Fig. 7 Morphodynamic bed equilibria are shown as solid red lines, together with the corresponding sediment transport contributions: diffusion (solid blue line), advection due to internal processes (dashed blue line) and advection due to external processes (dashed-dotted blue line). The embayment has a converging geometry. In a The relative phase is taken to be $\phi=195^{\circ}$, in b the relative phase is $\phi=67^{\circ}$

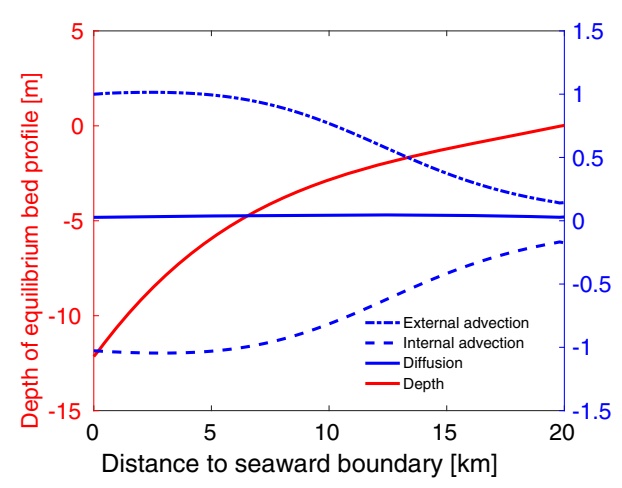

(a)

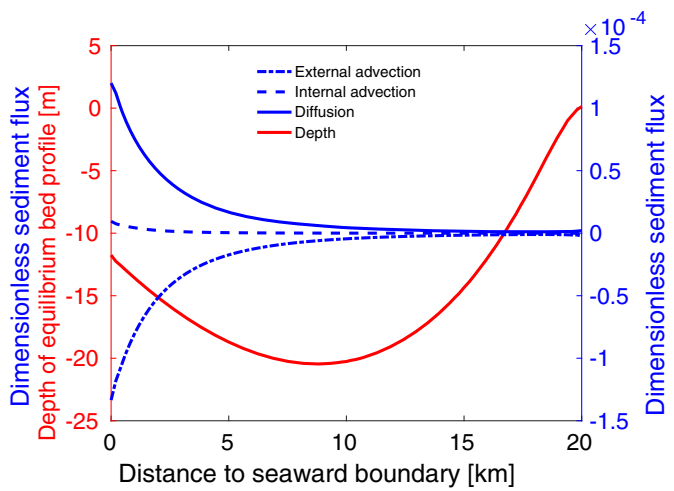

(b) 
where no equilibrium is found for larger lengths still exists, although it is much smaller than for a converging and rectangular embayment.

A new aspect is that the morphodynamic equilibria are not necessarily unique anymore; there exists a range of values for the relative phase and the embayment length for which multiple morphodynamic equilibria exist, see Fig. 8.

To study this in more detail, the mean water depth is plotted in Fig. 8b. Considering the embayment lengths $L=14 \mathrm{~km}$ and $L=18 \mathrm{~km}$, the mean depth of the morphodynamic equilibrium bed profile is large for $0^{\circ}<$ $\phi<180^{\circ}$ and changes to a shallow profile for a relative phase between $180^{\circ}<\phi<360^{\circ}$. For an embayment with $L=18 \mathrm{~km}$, this transition between a deep and a shallow equilibrium profile is more sudden than for $L=14 \mathrm{~km}$. For $L=19.6 \mathrm{~km}$ a saddle-node bifurcation takes place at approximately $\phi=70^{\circ}$ and $\phi=170^{\circ}$, respectively. In between these values of $\phi$, there exist multiple stable solutions, one of them corresponding to a deep profile and the other one to a more shallow character. The deeper equilibrium still exists when increasing the relative phase $\phi$ and the more shallow one when decreasing the relative phase. There is also a third unstable equilibrium with a shallow character.

In Fig. 8c, we give the three different bed equilibria that exist at a relative phase of $\phi=163^{\circ}$ for an embayment length of $L=19.6 \mathrm{~km}$. The corresponding sediment transport of these three equilibria are shown as a function of the location in the embayment in Fig. 9. We see for all three equilibria that the diffusive transport is very small and that the main balance exists between the internal and external advective sediment transport. Note that the sediment transport corresponding to the shallow bed profiles, Fig. 9b, c, look similar. When the bed profile is shallow, the sediment transport due to external advection is exporting, whereas it is importing when the bed profile has a deep character. The tidal embayment is changing from flood dominant to ebb dominant although the relative phase has

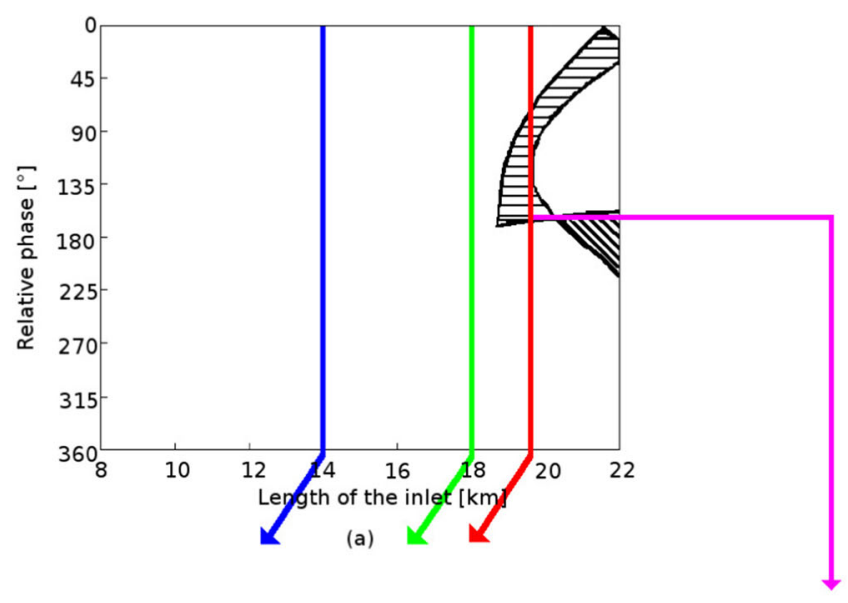

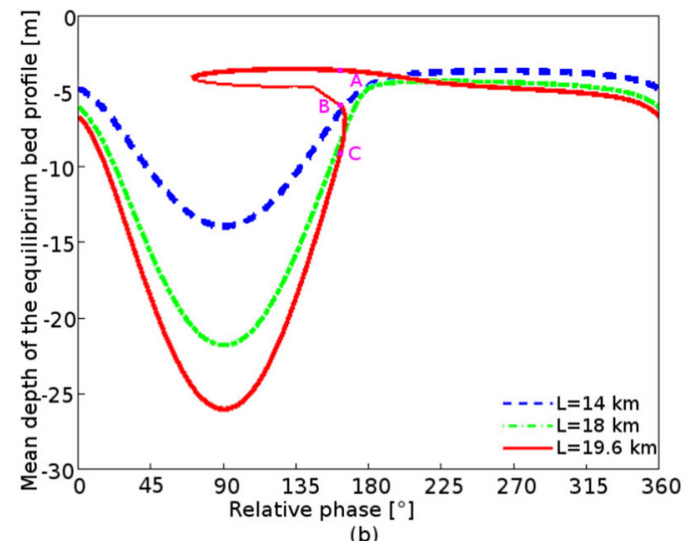

Fig. 8 a Plot of the number of equilibria for the relative phase versus the embayment length. For every combination in this parameter plane, the figure indicates whether there exists zero (diagonal lines), one (no lines) or multiple (horizontal lines) morphodynamic equilibria. b The mean value of the equilibrium bed profile is given versus the relative phase for three different embayment lengths $L=14 \mathrm{~km}$ (dashed

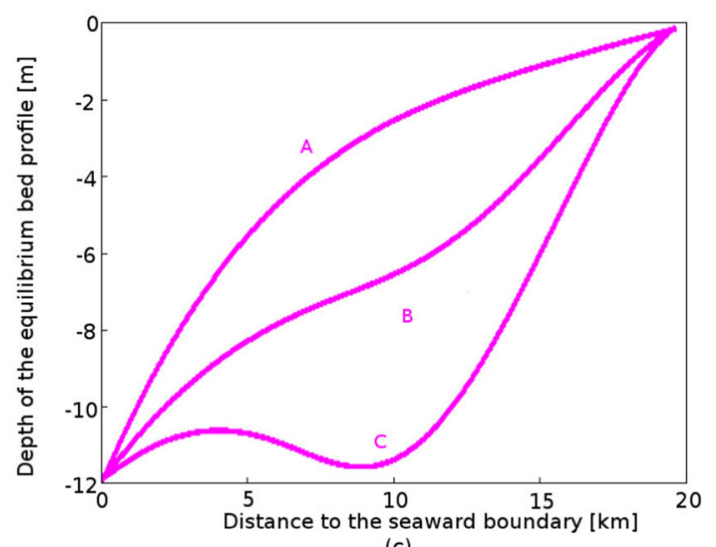

(c)

blue line), $L=18 \mathrm{~km}$ (dashed-dotted green line) and $L=19.6 \mathrm{~km}$ (red solid line). $\mathbf{c}$ The three different morphodynamic bed profiles are shown for a relative phase of $\phi=163^{\circ}$ and $L=19.6 \mathrm{~km}$. Here the points $\mathrm{A}, \mathrm{B}$ and $\mathrm{C}$ of $\mathbf{b}$ and $\mathbf{c}$ correspond to each other 
Fig. 9 The sediment transport corresponding to the three morphodynamic equilibria for an embayment length of $L=19.6 \mathrm{~km}$ and relative phase $\phi=163^{\circ}$. The subfigures $\mathbf{a}, \mathbf{b}$ and $\mathbf{c}$ correspond to the bed profiles A, B and C of Fig. 8c, respectively

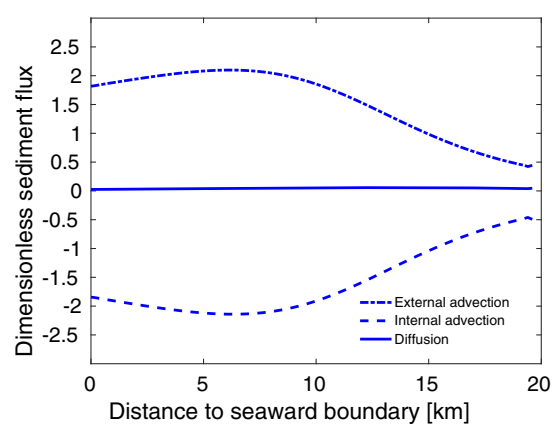

(a)

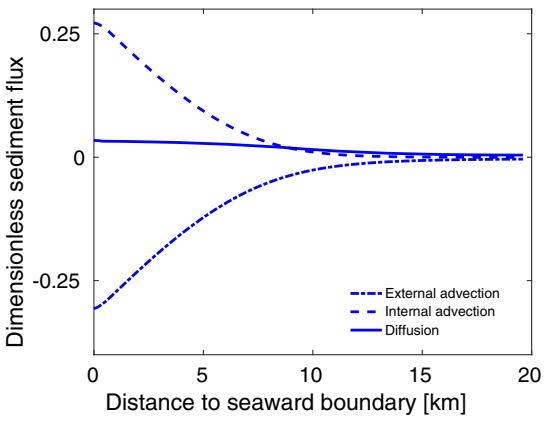

(b)

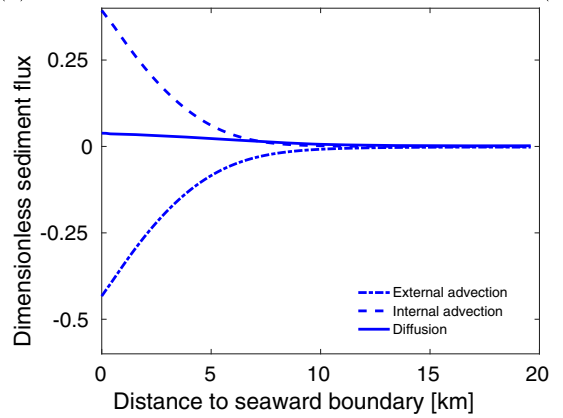

(c)

not been changed. When the geometry is more diverging, the values of the embayment length for which multiple equilibria exist decreases.

\subsection{Comparison with observed bed profiles}

In the previous subsections, the geometry of the embayment was taken to be either exponentially converging, exponentially diverging or rectangular. Since it is possible to choose arbitrary width variations in the model, we take a realistic width profile.

We have used data from observations of the Ameland Inlet and the Friesche Zeegat, both systems in the Dutch part of The Wadden Sea, with the water level measured at Nes and Schiermonnikoog.

Using observations of the depth, (2011 observations for the Ameland Inlet and 1987 for the Friesche Zeegat), we have determined a width profile and corresponding widthaveraged bed profile of each system. First, a depth profile of the area is constructed using measurements, and the center line through the inlet has been constructed. For points on the center line, perpendicular lines have been drawn from one lateral boundary to the other, where we defined the lateral boundary of the inlet at the locations where the undisturbed water was $0.5 \mathrm{~m}$. For each point, we choose the width of the embayment to be equal to the distance between the two coastlines. The corresponding depth is obtained by averaging the depth between the two coastlines. These width and depth profiles are shown by the dashed lines on the left side of Fig. 10 in blue and red, respectively.
We have then smoothed the obtained width profile and used this profile in our model to determine the corresponding morphodynamic equilibrium bottom. For the Ameland Inlet, we have used the characteristic values listed in Table 1 and for the Friesche Zeegat, the values used in Ter Brake and Schuttelaars (2010) have been employed. Furthermore, we have performed a harmonic analysis on the measured values of the water level at Nes and Schiermonnikoog resulting in the amplitude of the M2-tide and the M4-tide. A comparison between the data and the model results is presented in Fig. 10, showing a reasonable agreement with the main trends rather well-captured.

Concerning the amplitudes of the sea surface elevation, the difference between the observed and the modelled amplitudes varies from 3 to $10 \mathrm{~cm}$.

The difference between the data and the model results can have multiple reasons. One, it is unknown whether the characteristic values used in this article were the best choices for the physical parameters when the measurements were carried out. We have shown in the previous section that varying physical parameters can have a significant influence on the bed level and, hence, on the amplitudes of the tidal constituents. Second, the data amplitudes have been determined using measurements performed over a whole year, whereas the width and depth values have been obtained at one moment. Third, the measuring locations for the sea surface elevation are close to the coast. It is therefore not necessarily a representative value when comparing to the modelled width-averaged sea surface elevation. Furthermore, lateral processes are only parametrically taken 

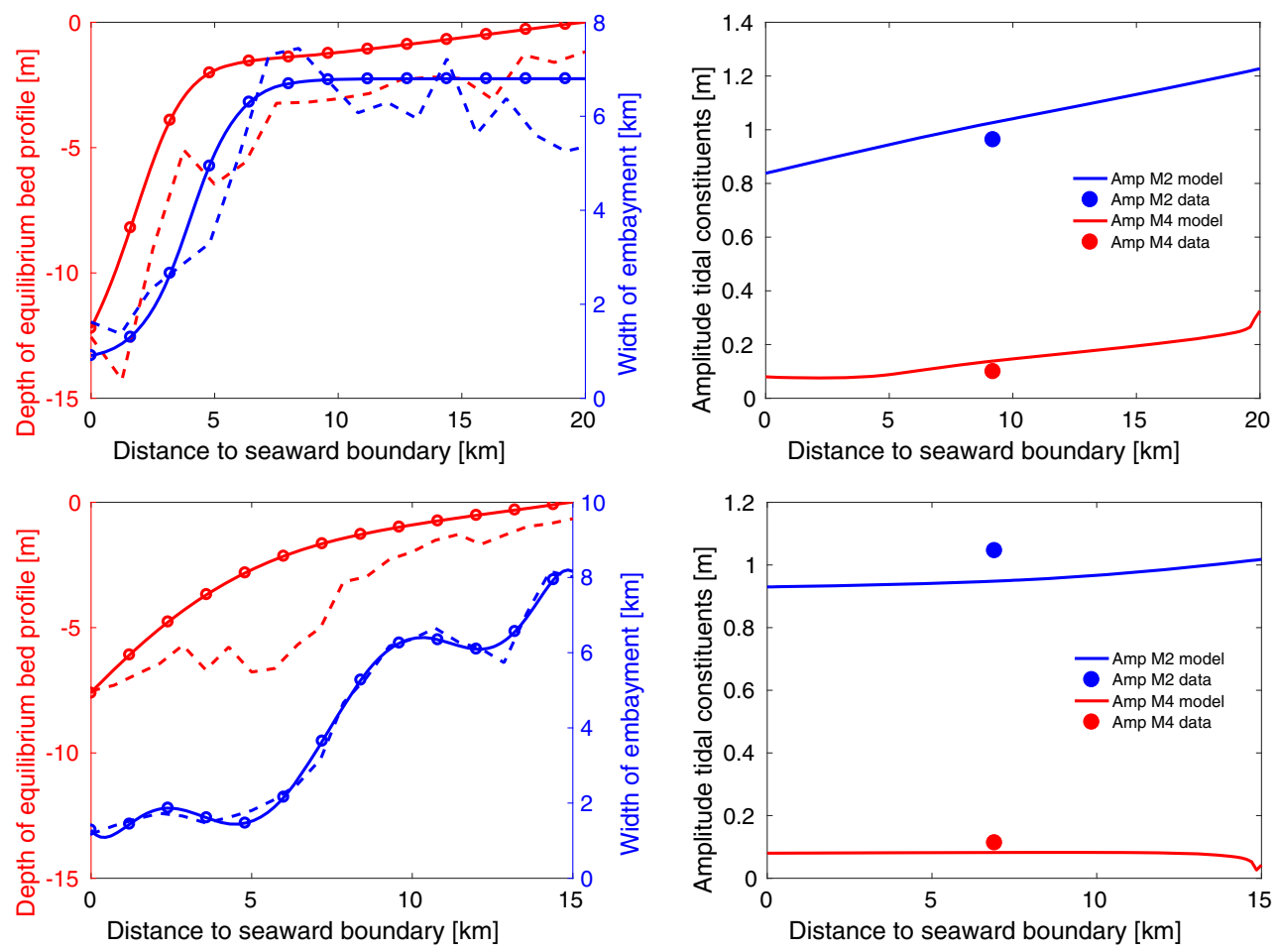

Fig. 10 A comparison between data and model results for the Ameland Inlet (top) and the Friesche Zeegat (bottom). At the left, the width profiles and the corresponding width-averaged bed profiles are shown as a function of the position in the embayment. The dashed lines represent the observed profiles and the solid lines the modelled profiles. At the right, the modelled amplitudes of the $M_{2}$ (blue lines) and the $M_{4}$ (red lines) tidal constituent are shown as a function of the position in the

embayment. The dots correspond to the values of the measured components. The measuring location Nes is about $7 \mathrm{~km}$ from the seaward boundary and the location Schiermonnikoog about $9.5 \mathrm{~km}$. Characteristic values for the Ameland Inlet are given in Table 1. For the Friesche Zeegat, the characteristic values that are different compared to the values of the Ameland Inlet are $A_{M_{2}}=0.93 \mathrm{~m}, \phi=207^{\circ}, H=7.5 \mathrm{~m}$ and $L=15 \mathrm{~km}$, see Ter Brake and Schuttelaars (2010)

into account, and many possibly important processes were not accounted for.

\section{Conclusion}

We have analysed the existence of morphodynamic equilibria and their sensitivity to geometrical variations, inertia, bottom friction, depth-dependency of the deposition parameter and the importance of advective processes both with and without an externally prescribed overtide. We considered tidally dominated embayments with a length between $L=8 \mathrm{~km}$ and $L=22 \mathrm{~km}$, representative for basins in the Wadden Sea. The geometry of the embayment was chosen to be rectangular, exponentially converging or exponentially diverging. We used the cross-sectionally averaged shallow water equations to describe the water motion and an advection-diffusion equation for the sediment concentration equation. We assumed that the bed evolves due to convergences and divergences of diffusive and advective sediment transport.

We started with only considering diffusive processes and prescribing the sea surface elevation by only a $M_{2}$-tide. For the frictionless case, the obtained morphodynamic bed equilibrium was highly influenced by varying the geometry. For a rectangular embayment, a constantly sloping bed profile was found. When the geometry was changed, the bed profile became more convex (concave) for a diverging (converging) embayment. Letting the deposition parameter depend on the depth resulted in more concave bed profiles. If we increased the embayment length, the frictional influence became stronger as well. The effect of bottom friction for a converging embayment was negligible, but becomes more significant for a more strongly diverging embayment. When also advective processes were taken into account, the influence of frictional effects increased significantly. When we considered diffusive and advective sediment transport without an externally prescribed overtide, we found that the bed profiles became less convex compared to those with only diffusive processes considered. Although this occurred for all geometries considered, the influence of adding advective sediment 
transport was much stronger when the geometry of the embayment was diverging.

When the prescribed sea surface elevation consisted of both a $M_{2}$ and a $M_{4}$ tide, we found a maximum length of the embayment for which there still existed morphodynamic equilibria. The maximum embayment length decreased when the geometry became more converging. The smallest embayment length was found for a relative phase of $\phi=$ $270^{\circ}$ for converging embayments. Making the geometry less converging, increasing the bottom friction or decreasing the amplitude of the $M_{4}$-tide, resulted in increasing maximum embayment lengths. We obtained two types of bed profiles, depending on the values of the relative phase: a shallow one where the external sediment transport was exporting and a deep one for importing external sediment transport.

When considering a diverging embayment geometry, there is a parameter range of relative phases and embayment lengths for which two stable bed profiles co-exist, along with a third unstable equilibrium. These results indicate that for systems with the characteristics of the Wadden Sea systems, there are parameter values for which both a relatively shallow and a much deeper width-averaged morphodynamic equilibrium can exist. For a proper management, it is important to be aware of the existence of multiple equilibria in tidal inlet systems. The analysis given in this paper does not indicate the magnitude of the perturbation necessary to go from one equilibrium to the other, nor the time scale at which a change would take place, this is topic of further research. However, we would like to stress that the existence of multiple equilibria has been observed in Schuttelaars and de Swart (2000) as well; they found multiple equilibria for a rectangular long embayment with a length of approximately $L=120 \mathrm{~km}$, these model results were confirmed by simulations done with a complex numerical model (Hibma et al. 2003). It would be interesting to investigate the presence of multiple equilibria for longer systems with width variations. For a rectangular system, this has already been done in Schuttelaars and de Swart (2000), where indeed it was found that multiple equilibria can exist as well (see Fig. 6 in that paper), and these results were qualitatively reproduced in the paper of Hibma et al. (2003) using a complex model.

The morphodynamic model derived in this paper can be improved upon by explicitly considering lateral processes what will result in observed complex channelshoal patterns. Furthermore, the accuracy of the water motion close to the landward boundary can be improved by considering more tidal harmonics, which might be necessary as the parameter $\epsilon$ is not small near this boundary. This might shed some light on the fact that we obtain equilibria which exist on a long morphodynamic timescale while many simulation models, like Van Ledden et al. (2004), and Maan et al. (2015) result in an infilling of embayments which occur on an even longer timescale. Another interesting extension to the existing model would be the inclusion of wind, waves and density flows, which have been shown to be important (Green and Coco 2014; Gatto et al. 2017; Burchard et al. 2008) and the inclusion of flooding and drying processes.

Funding information This research was supported by a grant of the NDNS+ cluster of the Dutch Science Organisation (NWO).

Open Access This article is distributed under the terms of the Creative Commons Attribution 4.0 International License (http:// creativecommons.org/licenses/by/4.0/), which permits unrestricted use, distribution, and reproduction in any medium, provided you give appropriate credit to the original author(s) and the source, provide a link to the Creative Commons license, and indicate if changes were made.

\section{References}

Burchard H, Flöser G, Staneva JV, Badewien TH, Riethmüller R (2008) Impact of density gradients on net sediment transport into the wadden sea. J Phys Oceanogr 38:566-587

Burchard H, Schuttelaars HM, Ralston DK (2018) Sediment trapping in estuaries. Annu Rev Mar Sci 10:371-395

Csanady GT (1982) Circulation in the coastal ocean reidel

De Swart HE, Zimmerman JTF (2009) Morphodynamics of tidal inlet systems. Annual Rev Fluid Mech 41:203-229

De Vriend HJ, Ribberink JS (1996) Mathematical modelling of meso-tidal barrier island coasts. Part II: process-based simulation models. In: Liu PLF (ed) Advances in coastal and ocean engineering, vol 2. World Scientific Publ Cy, pp 151-197

Dijkstra HA, Wubs FW, Cliffe AK, Doedel E, Dragomirescu IF, Eckhardt B, Gelfgat AY, Hazel AL, Lucarini V, Salinger AG, Phipps ET, Juan S-U, Schuttelaars H, Tuckerman LS, Thiele U (2014) Numerical bifurcation methods and their application to fluid dynamics: analysis beyond simulation. Commun Comput Phys 15:1-45

Dyer KR (1986) Coastal and estuarine sediment dynamics. Wiley, Chichester

Friedrichs CT (2010) Contemporary issues in estuarine physics. Cambridge University Press, Cambridge

Friedrichs CT, Aubrey DG (1996) Uniform bottom shear stress and equilibrium hypsometry of intertidal flats, in mixing in estuaries and coastal seas. Coast Estuar Stud 50:405-429

Gatto VM, Van Prooijen BC, Wang ZB (2017) Net sediment transport in tidal basins: quantifying the tidal barotropic mechanisms in a unified framework. Ocean Dyn 67:1385-1406

Glaeser DJ (1978) Global distribution of barrier islands in terms of tectonic setting. J Geol 86:283-297

Green G (1837) On the motion of waves in a variable canal of small depth and width. Trans Cambridge Pilos Soc 6:457-163

Green MO, Coco G (2014) Review of wave-driven sediment resuspension and transport in estuaries. Rev Geophys 52:77117

Hibma A, Schuttelaars HM, Wang ZB (2003) Comparison of longitudinal equilibrium profiles of estuaries in idealized and process-based models. Ocean Dyn 53:252-269

Lanzoni S, Seminara G (1998) On tide propagation in convergent estuaries. J Geophys Res 103:30793-30812

Lanzoni S, Seminara G (2002) Long-term evolution and morphodynamic equilibrium of tidal channels. J Geophys Res 107:1-13 
Maan DC, Prooijen BC, Wang ZB, De Vriend HJ (2015) Do intertidal flats ever reach equilibrium? J Geophys Res 120:240 2436

Murray AB (2013) Contrasting the goals, strategies, and predictions associated with simplified numerical models and detailed simulations. Am Geophys Union, pp 151-165. https://doi.org/10.1029/ 135GM11

Parker BB (ed) (1991) Tidal hydrodynamics. Wiley, New York

Pritchard D, Hogg AJ (2003) Cross-shore sediment transport and the equilibrium morphology of mudflats under tidal currents. $\mathrm{J}$ Geophys Res 108:2156-2202

Ridderinkhof H, Zimmerman JTF (1992) Chaotic stirring in a tidal system. Science 258:1107-1111

Ridderinkhof W, Swart HE, Vegt M, Hoekstra P (2016) Modeling the growth and migration of sandy shoals on ebb-tidal deltas. J Geophys Res Earth Surf 121:1351-1375

Robert P, Le Hir W, Whitehouse RJS (2000) Investigation using simple mathematical models of the effect of tidal currents and waves on the profile shape of intertidal mudflats. Cont Shelf Res 20:1079-1097

Schuttelaars HM, de Swart HE (1996) An idealized long-term morphodynamic model of a tidal embayment. Eur J Mech B Fluids 15:55-80

Schuttelaars HM, de Swart HE (2000) Multiple morphodynamic equilibria in tidal embayments. J Geophys Res 105:24, 105-24, 118

Seminara G, Lanzoni S, Tambroni N, Tofffolon M (2010) How long are tidal channels. J Fluid Mech 643:479-494

Smith JD, McLean SR (1977) Spatially averaged flow over a wavy surface. J Geophys Res 82:1735-1746
Ter Brake MC, Schuttelaars HM (2010) Modeling equilibrium bed profiles of short tidal embayments. Ocean Dyn 60:183-204

Todeschini I, Toffolon M, Tubino M (2008) Long-term morphological evolution of funnel-shape tide-dominated estuaries. J Geophys Res, 113: https://doi.org/10.1029/2007JC004094

Toffolon M, Lanzoni S (2010) Morphological equilibrium of short channels dissecting the tidal flats of coastal lagoons. J Geophys Res 115:2156-2202

Van der Wegen M, Jaffe B, Foxgrover A, Roelvink D (2017) Mudflat morphodynamics and the impact of sea level rise in South San Francisco bay. Estuar Coasts 40:37-49

Van Ledden M, Wang ZB, Winterwerp H, De Vriend H (2004) Sand-mud morphodynamics in a short tidal basin. Ocean Dyn 54:385-391

Van Leeuwen SM (2002) Tidal inlet systems: bottom pattern formation and outer delta development. $\mathrm{PhD}$ thesis, Universiteit Utrecht

Van Leeuwen S, Schuttelaars H, de Swart HE (2000) Tidal and morphologic properties of embayments: effect of sediment deposition processes and length variation. Phys Chem Earth B 25:365-368

Van Prooijen BC, Wang ZB (2013) A 1d model for tides waves and fine sediment in short tidal basins - application to the wadden sea. Ocean Dyn 63:1233-1248

Van Rijn LC (1993) Principles of sediment transport in rivers, estuaries and coastal seas. Aqua Publishing, Amsterdam

Zimmerman JTF (1981) Dynamics, diffusion and geomorphological significance of tidal residual eddies. Nature 290:549-555

Zimmerman JTF (1992) On the Lorentz-linearization of a nonlinearly damped tidal Helmholtz oscillator. Proc Kon Ned Akad v Wetensch 95:127-145 